\title{
GT2019-91421
}

\section{INVESTIGATING ETHANOL-GASOLINE SPRAY CHARACTERISTICS USING AN INTERFEROMETRIC DROP SIZING TECHNIQUE}

\author{
Hywel Davies \\ Department of Mechanical Engineering \\ University College London \\ London WC1E 7JE, UK \\ hywel.davies.14@ucl.ac.uk \\ Martin Hyde \\ TSI Instruments Ltd. \\ Cressex Business Park \\ High Wycombe HP12 3ST, UK \\ mhyde@tsi.com
}

\author{
Midhat Talibi * \\ Department of Mechanical Engineering \\ University College London \\ London WC1E 7JE, UK \\ m.talibi@ucl.ac.uk
}

\author{
Ramanarayanan Balachandran \\ Department of Mechanical Engineering \\ University College London \\ London WC1E 7JE, UK \\ r.balachandran@ucl.ac.uk
}

\section{ABSTRACT}

To reduce reliance on fossil fuels there has been a global push to minimise fuel consumption, and incorporate the use of bio-derived fuels. In practical combustion systems that use liquid fuels, observing the spray behaviour of these biofuels is key in understanding fuel performance; in particular, droplet size distribution is known to have a strong influence on the fuel energy release and pollutant formation processes. This paper is aimed at the use of the TSI Global Sizing Velocimetry (GSV) interferometric technique as a method to gain detailed understanding of droplet number and size distribution, with a particular focus on ethanol-gasoline fuel blends.

The imaging system for the GSV technique consisted of a Nd:YAG laser and a $4 \mathrm{MP}$ (million pixel) camera, and the spray was generated using a generic automotive port fuel injector. The results showed that the GSV technique was able to effectively measure droplet concentration and diameters for all the fuel blends tested in this study. Ethanol was observed to have larger droplet diameters (both $D_{10}$ and $D_{32}$ ) as compared to fossil gasoline, with droplet diameters generally increasing as the proportion of ethanol in the gasoline was increased. The droplet concentration reduced with increasing radial distance from the spray centreline, but no appreciable change was observed with axial displacement from the nozzle tip. Degradation in the image quality was observed for fuel blends with less than $40 \%$ ethanol content. The GSV drop sizing measurements were validated using a mono-disperse droplet generator, and an excellent agreement (within 2\%) was observed.

\section{NOMENCLATURE}

GSV Global Sizing Velocimetry

HSI Horizontal Scan Interval

MPSPR Minimum Power Spectrum Peak Ratio

PFI Port Fuel Injection

PDPA Phase Doppler Particle Anemometry

VSO Vertical Scan Output

$D \quad$ Individual Droplet Diameter Readings

$D_{10} \quad$ Arithmetic Mean

$D_{32}$ / SMD Sauter Mean Diameter

$\mathrm{X} \quad$ Radial Displacement

Y Axial Displacement 


\section{INTRODUCTION}

In every application of liquid spray, be it spray drying in pharmaceutics, spray cooling in nuclear reactors or fuel injection in combustion engines, understanding spray characteristics enables a more informed nozzle design and allows optimisation of operating conditions. For example, a main governing factor in the combustion of liquid fuel sprays is the degree of atomisation and the resulting size of fuel droplets. For a fixed volume of fuel, a dispersed distribution of smaller droplets creates a more homogenous air fuel mixture (which results in more uniform combustion), as compared to fewer larger droplets.

Techniques such as Phase Doppler Particle Anemometry (PDPA), ratiometry and interferometric droplet sizing techniques are known for measurements of droplet diameters [1-6]. Pierson et al. [1] used PDPA to characterise spray from a multi-hole gasoline port fuel injector, with the aim of using the findings as validation for computational spray modelling. Moriyoshi et al. [6] successfully measured the influence of crossflow on droplet size, velocity and the droplet distribution in direct injection gasoline sprays using interferometric droplet sizing techniques. An advantage of planar sizing techniques, such as interferometric droplet sizing techniques, over point measurement techniques, such as PDPA, is the increased field of view (typically $10 \times 10$ $\mathrm{mm}$ ) [7], which allows for a better understanding of the global spray properties, and expedites the process of building a complete picture of droplet sizes across the entire spray.

Increasing concerns regarding the environmental impact of fossil fuel combustion has led to many governing bodies mandating the use of sustainable bio-derived fuels. Bio-ethanol is one of the most commonly used bio-fuels due to its miscibility in gasoline, its higher octane number and its well understood production from plant matter. Understanding spray formation and dynamics of such bio-fuels is fundamental for the development of more efficient combustion systems with reduced pollutant emissions. Park et al. [8] investigated blends of bio-ethanol and gasoline using PDPA, and reported an increase in droplet diameters with increasing proportion of ethanol. Aleiferis et al. [5] found the same trends in droplet diameter with ethanol addition in fossil gasoline, when analysing spray behaviour from spark-eroded and laser-drilled injectors using PDPA.

It is evident from literature that the interferometric droplet sizing technique has excellent potential for detailed spray analysis, however, it has not previously been used for the study of ethanol fuel blends, particularly relevant for propulsion applications, with most researchers using point measurement techniques for fuel blends. This paper investigates the effect of ethanol content in fossil gasoline on droplet diameters using the commercial interferometric drop sizing system, Global Sizing Velocimetry (GSV), developed by TSI Instruments Ltd. The specific objectives of this paper are to investigate i) the applicability of the GSV technique in measuring the droplet diameters of ethanolgasoline fuel blends, ii) the capability of the technique to deter- mine the spatial distribution of droplet number and size and iii) variations in droplet size for different blends of ethanol and gasoline. The structure of the paper is as follows; a brief description of the TSI GSV system has been provided, followed by the experimental methodology, data reduction and analysis, key results and discussion, and conclusions and future work.

\section{Experimental Methodology}

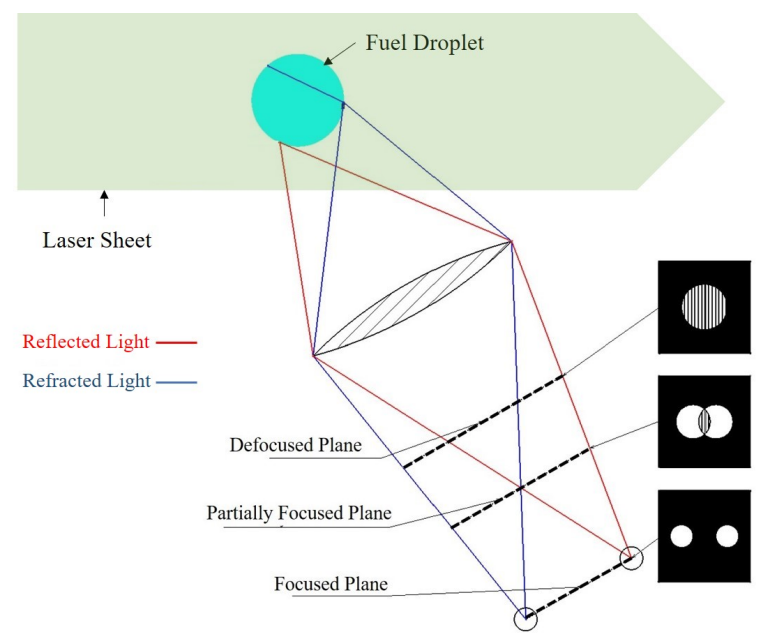

FIGURE 1. DIAGRAM SHOWING INTERFEROMETRIC PRINCIPLES

\subsection{Principle of interferometric techniques}

Interferometric droplet sizing techniques use both the reflected and refracted images from droplets to accurately determine their diameters, as can be seen in Fig. 1. The camera is set at a particular angle to the laser plane in order capture both reflected and refracted images. The camera is then deliberately defocused, so that the images superpose, creating an interference pattern. The number of oscillations and the fringe spacing of this pattern are used to determine the droplet size.

\subsection{TSI Instruments GSV system}

The TSI Instruments GSV setup uses the same basic principles of interferometric droplet sizing techniques described above. The imaging system for the GSV technique consists of a pulsed Nd:YAG laser (Quantel Evergreen 200) and a 4 MP (TSI PowerView, 12 bit, 2352 x 1768 pixels) camera. The laser sheet thickness was set to roughly $250 \mu \mathrm{m}$ using variable focus optical lenses, with the laser energy being $185 \mathrm{~mJ}$ (pulse-width of 10 $\mathrm{ns})$. A $100 \mathrm{~mm}$ focal length lens was used with the aperture set 


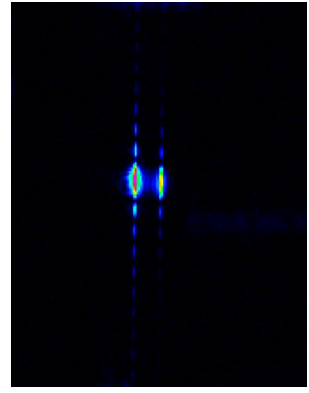

(a)

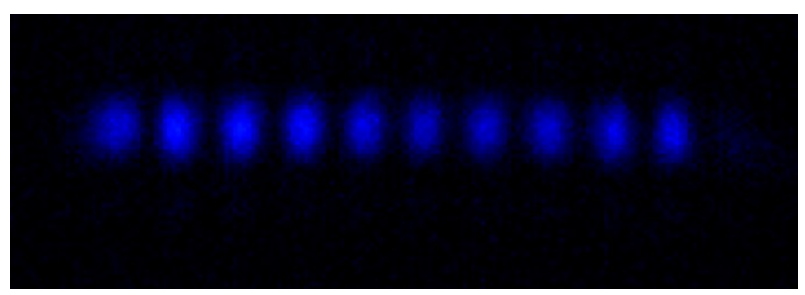

(b)

FIGURE 2. (a) GLARE POINTS AT FOCUS, (b) INTERFERENCE OSCILLATION WITH SLIT APERTURE AT $8.55 \mathrm{~mm}$ DEFOCUS.

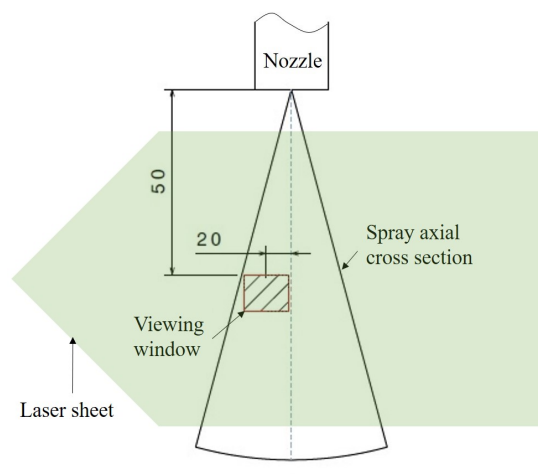

FIGURE 3. SIDE VIEW DIAGRAM OF SPRAY CROSS SECTION WITH SELECTED VIEWING WINDOW.

to F5.6 for the all the tests. The lens was focused at its minimum focal distance of $300 \mathrm{~mm}$ in order to produce a 1:1 magnification ratio, giving the best possible spatial resolution. The camera exposure time was set to $1 \mathrm{~ms}$. The laser and camera were mounted such that their planes were at $60^{\circ}$ to each other, for which the GSV software is optimised. Due to this angle between the laser and camera focus planes, only the centre of the image was set in focus. Initially, the focus of the camera was adjusted so that the droplets along the vertical centreline of the viewing window appeared as double glare points. An example of this is shown in Fig. 2(a). The camera was then moved away from its focused position to create interference of the reflected and refracted images and form oscillation patterns, as can be seen in Fig. 2(b). Droplet

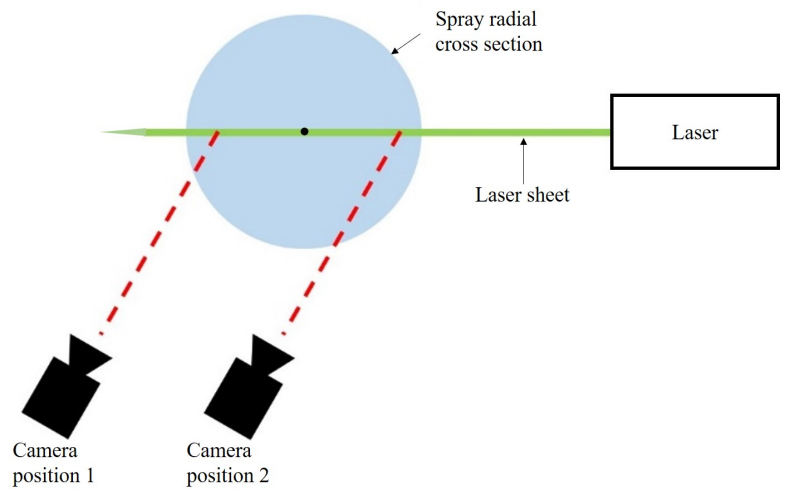

FIGURE 4. TOP VIEW DIAGRAM OF SPRAY CONE SECTION, WITH (CAMERA POSITION 1) LEFT SIDE IMAGING AND (CAMERA POSITION 2) RIGHT SIDE IMAGING.

diameters are determined from the spacing between the fringes within these patterns. The required amount of defocusing necessary is based on two main factors, the clarity of individual oscillation patterns and the degree of overlap between multiple oscillation patterns. If the image is not sufficiently defocused, oscillation patterns become too compact making the spacing between fringes difficult to measure accurately. This hampers the precision of the droplet diameters measured. Excessive defocusing results in drawn out, non-distinct and lower intensity oscillation patterns making it similarly difficult to determine the spacing between fringes. This also increases the degree of overlap between oscillation patterns due to their larger size. As mentioned earlier, the camera and laser planes are offset by $60^{\circ}$, as such one side of the image is closer to the camera than the other and so is less defocused than the other. The TSI software corrects for this disparity when analysing the oscillation patterns, but it is important to ensure that the oscillation patterns in the more defocused portion of the image are not too drawn out and of very low intensity, and that the oscillations in the less defocused region are not too compact. It is recommended that the oscillation patterns in the less defocused region of the image should be at a minimum 96 pixels in length. In line with this recommendation, a minimum oscillation pattern length of 110 pixels was kept for all the experimental conditions. To get the required interference pattern, for these experiments, the camera was defocused by moving it $8.55 \mathrm{~mm}$ away from the laser plane, and was fixed for the entirety of experimentation. A micrometre-controlled traverse was used to get precise measurement of the defocus displacement. A slit aperture was mounted on the camera lens, which compressed the interference pattern from a full circle to thin single line of fringes, as can be seen in Fig. 2(b). This enabled the GSV software to analyse denser sprays without having oscillation patterns overlapping and causing erroneous readings of droplet diameters.

The GSV image analyser software uses a windowed fast 
Fourier transform (FFT) technique to identify the frequency of the oscillation patterns. The basic principle of this technique is that strong oscillations show a single dominant frequency, whilst oscillation patterns created by invalid droplets, such as non-spherical droplets and background noise, exhibit multiple dominant frequencies. Once clear and valid oscillation patterns are identified, the spacing between fringes are analysed to determine the droplet size [7]. The ability to measure droplets is dependent on the presence of at least two fringes in an oscillation pattern. Using the current setup, the minimum droplet size that can be measured is $10 \mu \mathrm{m}$, any droplets smaller than this will not be detected.

A potential limiting factor when using any interferometric technique is that, in regions of high droplet density, oscillations overlap creating blurred or high frequency oscillations. As such, TSI recommend that the maximum droplet count per cubic centimetre in the viewing region should be 3000 . Preliminary tests were carried out by capturing different regions of the spray, in order to ensure that the droplet density within the selected viewing window was not above the recommended level. To measure the droplet density in the region of interest (ROI), a sample of 20 images was taken. Based on visual examination, the most densely populated quarter of each image was selected and analysed, with the total number of droplets contained within the quarter averaged over the 20 sample images. Using this value, the laser sheet thickness and ROI geometry, the droplet density per cubic centimetre was calculated. In the denser regions of spray, such as along the spray centreline and close to the injector nozzle, the droplet concentration was considerably above 3000 droplets per cubic centimetre, leading to droplet overlaps and a reduction in the number of valid readings. Axially and radially displacing the viewing window (ROI) from the nozzle tip reduced the concentration of droplets, which is concurrent with findings reported in previous literature $[3,9,10]$. In this study it was found that offsetting the viewing window $50 \mathrm{~mm}$ vertically from the nozzle and $20 \mathrm{~mm}$ radially from the spray centreline, shown in Fig. 3, meant the droplet concentration did not exceed the recommended limit.

The spray used in this study is symmetrical, which implies that either the left or right side of the spray cone can be imaged for analysis, as shown in Fig. 4. Viewing the right-side image was found to degrade the image quality and reduce the number of valid data readings as compared to the left side image. This is likely due to the increased volume of spray between the image and camera causing scattering of the light, known as off axis attenuation, resulting in a weaker signal reaching the camera sensor. As such the ROI selected for this study was located on the left-hand side of the spray centreline, shown in Fig. 3.

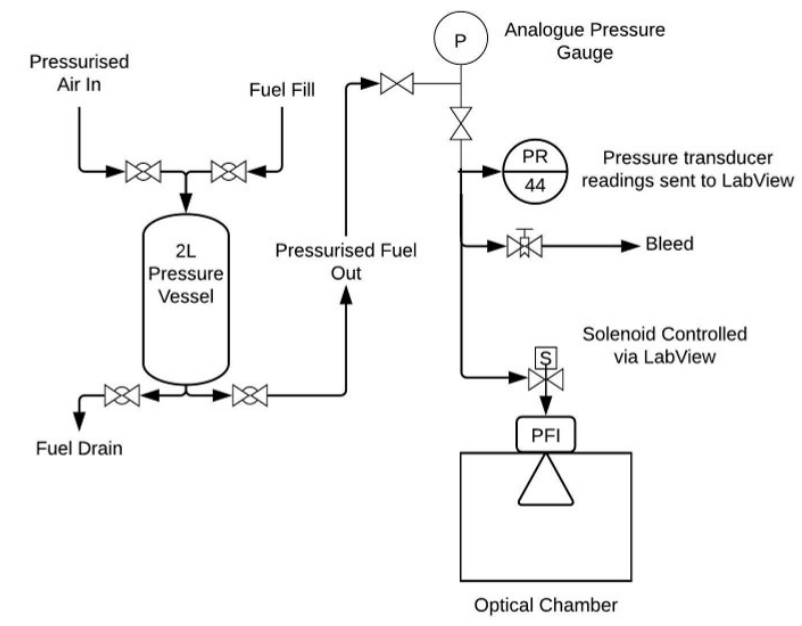

FIGURE 5. SCHEMATIC OF SPRAY TEST FACILITY (PPRESSURE GAUGE, PR-PRESSURE TRANSDUCER, PFI-PORT FUEL INJECTOR).

\subsection{Spray test facility}

Figure 5 shows the setup of test facility, including the fuel pressurisation and injection systems. An automotive, single hole, solid cone port fuel injector (PFI) was used to generate the spray. For all the tests, the fuel was injected at a pressure of 3.5 bar (flow rate of 0.73 litres per minute), which is within the PFI injector's specified operating range and demonstrated good atomisation during preliminary testing. The fuel was pressurised using a pressure vessel supplied with regulated pressurised air to achieve the desired fuel pressure. The fuel pressure was monitored by both an analogue gauge, and a digital pressure transducer which was monitored live on the LabVIEW interface using National Instruments data acquisition systems. The experiments were conducted in a test facility where the ambient temperature was maintained at a constant value for the duration of the tests. Spray timing and injection duration were controlled via LabVIEW, while the TSI Insight $4 \mathrm{G}$ software was used to time the camera and laser trigger signals. To contain the spray and to protect it from ambient effects, the injector was mounted in an optical vessel maintained at atmospheric pressure. Large viewing ports facilitated the required $60^{\circ}$ angle between the camera and laser planes. To ensure the spray was fully developed when the camera was triggered, various time delays between the injector and camera trigger were tested. It was found that there was no change in the droplet distribution with delays of $30 \mathrm{~ms}$ and greater. Therefore, the delay between the start of the injector trigger and the start of the camera trigger for all experiments was set to $40 \mathrm{~ms}$. The injector was opened for a total of $50 \mathrm{~ms}$. 


\subsection{Fuel blends}

To gain a comprehensive understanding of the effect of ethanol content on droplet diameters, a full range of fuel blends were tested, as can be seen in Tab. 1. It should be noted that the pure gasoline used had no ethanol content.

TABLE 1. FUEL BLENDS.

\begin{tabular}{c|c} 
Fuel blend & Ethanol content (\%) \\
\hline \hline E100 & 100 \\
E80 & 80 \\
E60 & 60 \\
E50 & 50 \\
E40 & 40 \\
E30 & 30 \\
E20 & 20 \\
E15 & 15 \\
E10 & 10 \\
E0 & 0 \\
\hline
\end{tabular}

\section{Data reduction and analysis}

This section will discuss the selection of processor parameters on the TSI Insight $4 \mathrm{G}$ software, in order to optimise the analysis of droplet sizing and identify the single most dominant frequency using the windowed FFT technique. For this purpose, preliminary tests were carried out in which E100 spray images were captured, and in the first instance, analysed with the default processor settings. Visual inspection of the analysed images revealed that some droplets were being incorrectly detected by the processor. Generally, these manifested in one of two forms: (1) Due to low intensity indistinct oscillation patterns, which generally lead to erroneous readings of low diameter droplets, as shown in Fig. 6(a). (2) Due to the additional interference in the oscillation pattern caused by multiple frequencies. Interferometric techniques ideally use the first refraction to create oscillation patterns. However, in practice higher order refractions are present, and in some circumstances these higher order refractions are refracted at such an angle that they are picked up by the camera, as found by Dehaeck et al. [11]. This results in the presence of multiple frequencies in the oscillation pattern, as is visible in Fig. 6(b). In some instances, oscillation patterns containing multiple frequencies are interpreted as a single higher frequency as shown in Fig. 6(c), resulting in the erroneous identification of

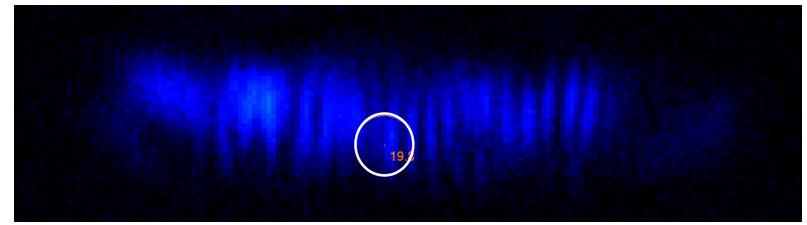

(a)

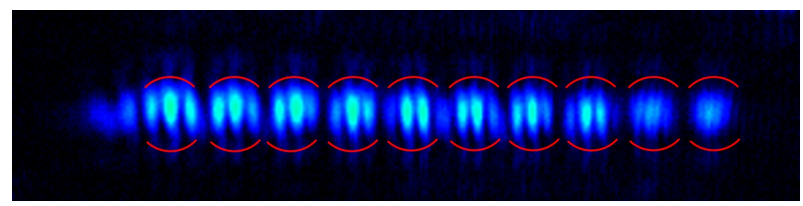

(b)

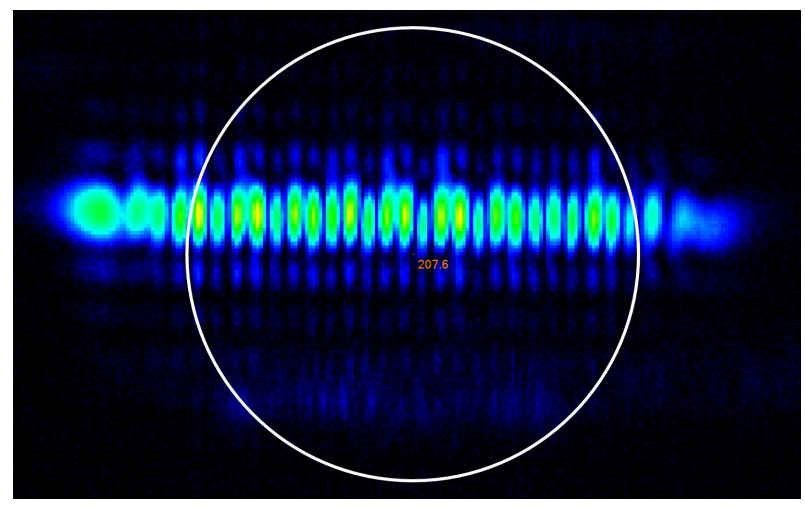

(c)

FIGURE 6. (a) LOW INTENSITY INDISTINCT OSCILLATION PATTERNS ERRONEOUSLY RECOGNISED AS SMALL DROPLET $(19.8 \mu \mathrm{m})$, (b) OSCILLATION PATTERNS SHOWING TWO FREQUENCIES, RED OUTLINE SHOWING THE FRINGES FROM ONE FREQUENCY, EACH CONTAINING 'SUB-FRINGES' FROM A SECOND FREQUENCY, (c) OSCILLATION PATTERNS CONTAINING MULTIPLE FREQUENCIES BEING ERRONEOUSLY MEASURED AS A LARGE DROPLET DIAMETER $(207.6 \mu \mathrm{m})$.

large diameter droplets. These high frequency oscillation patterns were also of higher intensity due to the additional light from multiple signals, as observed by Dehaeck et al. [11]. As well as the errors described, it was observed that not all clear oscillation patterns present in the image were being detected. The selection of the processor parameters was therefore focused on reducing the occurrence of low diameter and large diameter erroneous readings, as well as maximising the overall number of droplets detected from each captured image to reduce the total number of images required to produce a valid data-set.

Three particular parameters were observed to have the largest impact, Vertical Scan Output (VSO), Horizontal Scan Interval (HSI) and Minimum Power Spectrum Peak Ratio (MPSPR). VSO allows the selection of the method used to define a general spacing (either mean, median or maximum) between 


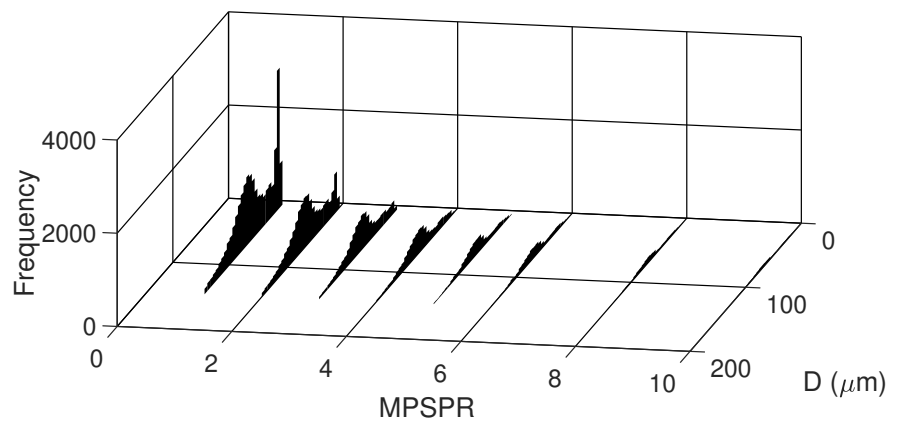

FIGURE 7. FREQUENCY DISTRIBUTION OF DROPLET DIAMETERS WITH MPSPR VARIATION.

fringes within an oscillation pattern. Whilst setting the VSO as maximum recorded the largest number of data points, it also produced a larger number of low diameter erroneous readings. The frequency of low diameter droplets was reduced (with no appreciable effect on the detection of large droplets) when using both the mean and median settings, with the mean setting showing the most reduction, hence this was chosen as the VSO setting for the current piece of work. The HSI parameter defines the minimum spacing, in pixels, between the fringes within an oscillation pattern. Increasing the HSI value beyond 20 significantly increased the number of low diameter readings, as did values below 10. Similar to the VSO, the detection of large diameters did not vary significantly with this parameter. A HSI value of 15 was selected for this study. The MPSPR defines the minimum signal to noise ratio of the analyser. Changing the MPSPR value had the largest influence on the droplet detection and diameter measurement. The MPSPR at its lowest value of 1 gave the highest droplet count, however however this had significant impact of increasing the low diameter peak, as can be seen on the histogram in Fig. 7. As the MPSPR value was increased above 1, the number of recorded droplets decreased, as did the frequency of low diameter droplets. The number of large droplet diameters also reduced when increasing the MPSPR value. As such, choosing the best value was a compromise between data count and the inclusion of potentially erroneous data. Based on the review of the data collected, a MPSPR value of 3 was set for the current set of experiments. The values of the three parameters were kept constant for the duration of the study.

Figure 8 shows the frequency and volume probability distribution for E100, obtained using the above mentioned optimised parameter settings. It can be seen from the figure, that a small number of large droplets (above $200 \mu \mathrm{m}$ ) contribute a considerable proportion to the overall volume of the spray. As stated previously, many of these large droplet diameters are due to oscillation patterns containing two dominant frequencies appearing as one higher frequency oscillation pattern (see Fig. 6c). Whilst this phenomenon is infrequent (as evidenced by the low propor-

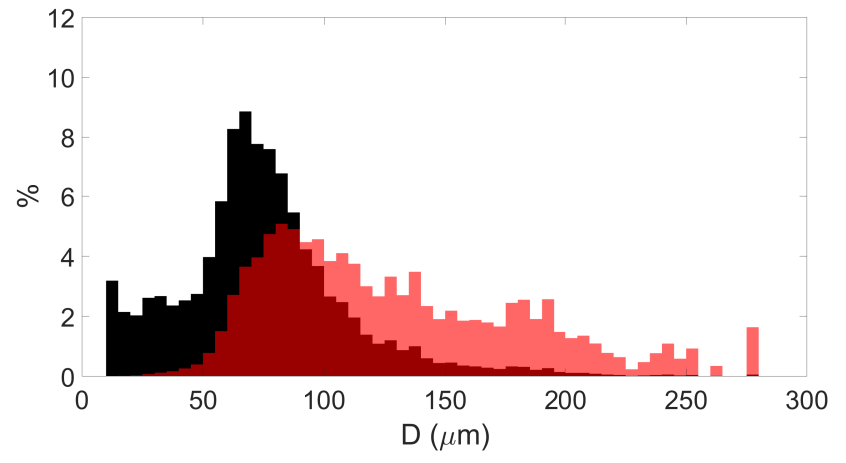

FIGURE 8. SUPERPOSED PROBABILITY DISTRIBUTIONS OF DROPLET NUMBER (BLACK) AND VOLUME (RED), EXPRESSED AS A PERCENTAGE OF OVERALL NUMBER AND TOTAL VOLUME RESPECTIVELY, FOR E100

tions of the larger droplets in Fig. 8), it could have an impact on the measurement of the average droplet diameters.

\section{Validation of GSV technique and measurement un- certainty}

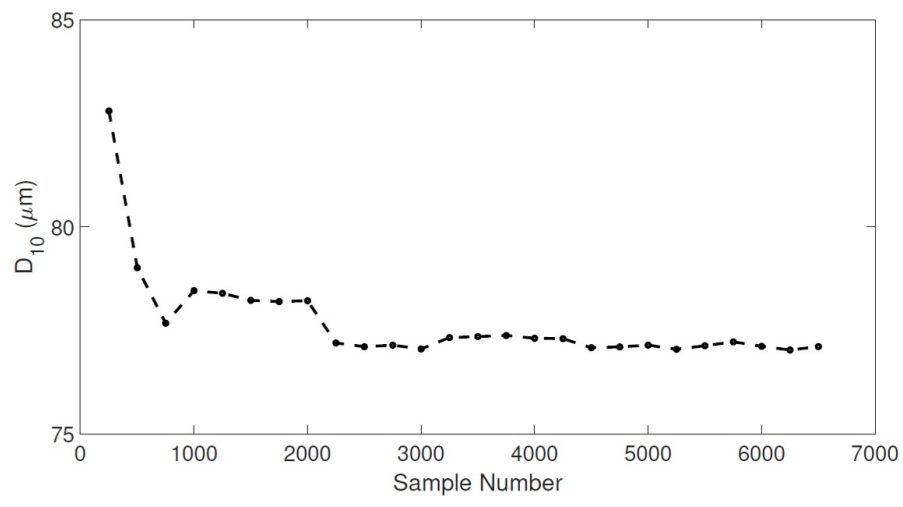

FIGURE 9. $D_{10}$ VARIATION WITH SAMPLE NUMBER

A droplet generator was used to validate the droplet sizing measurements obtained from the GSV setup. The droplet generator used was a Flow-Focusing Mono-disperse Aerosol Generator Model 1520 (FMAG), able to create droplets ranging between 15 and $90 \mu \mathrm{m}$ with a stated uncertainty of $\pm 1.0 \%$. Table 2 shows the comparison of the droplet sizes between the generator and GSV measurement. The four different diameters were chosen that represented the values around the peak diameters observed during this study. In all cases, the droplet size measured by GSV was in good agreement with the diameters produced by the droplet generator, the difference ranging between 0.3 and 1.6 
TABLE 2. COMPARISON OF DROPLET DIAMETERS FROM FLOW-FOCUSING MONO-DISPERSE AEROSOL GENERATOR AND GSV MEASUREMENT

\begin{tabular}{c|c|c|c}
$\begin{array}{c}\text { FMAG } \\
\text { Diameter } \\
(\mu \mathrm{m})\end{array}$ & $\begin{array}{c}\text { GSV } D_{10} \\
(\mu \mathrm{m})\end{array}$ & $\begin{array}{c}\text { Difference } \\
(\mu \mathrm{m})\end{array}$ & $\begin{array}{c}\text { Standard } \\
\text { Deviation } \\
(\mu \mathrm{m})\end{array}$ \\
\hline 71.3 & 72.4 & 1.1 & 1.5 \\
65.6 & 65.9 & 0.3 & 1.6 \\
62.0 & 63.5 & 1.5 & 1.5 \\
52.0 & 53.6 & 1.6 & 1.2 \\
\hline
\end{tabular}

TABLE 3. E100 AND E0 REPEAT TESTS.

\begin{tabular}{c|c|c} 
Blend & Initial $D_{10}(\mu \mathrm{m})$ & Repeat $D_{10}(\mu \mathrm{m})$ \\
\hline \hline E100 & 73.67 & 73.30 \\
E0 & 63.94 & 64.72 \\
\hline
\end{tabular}

$\mu \mathrm{m}$. The GSV results show a normal distribution of droplet diameter readings about the mean diameter value (see Fig. A1 in Appendix). The average standard deviation of the four test cases was $1.5 \mu \mathrm{m}$, which will be used to represent the measurement uncertainty in later analysis. In the work by Duan et al. [12] , the FMAG droplet sizes were measured using PDPA, and a standard deviation of $1.01 \mu \mathrm{m}$ was observed for droplet diameters above $40 \mu \mathrm{m}$. This is in good agreement with the standard deviation determined in the current piece of work.

The variation in the arithmetic mean of the droplet diameters, $D_{10}$, has been shown in Fig. 9 as a function of the number of droplets measured. The figure clearly shows that the variation in $D_{10}$ reduced to a minimum beyond 2500 droplet measurements for E100. Hence, for all the fuel blends the minimum sample size of droplets measured was no less than 5000 to ensure a valid data set size.

To ensure there were no significant changes in the laser or optical alignment during the experimentation period, the first two fuel blends tested (E100 and E0) were repeated at the end, and the results of this analysis have been tabulated, Tab. 3. As can be seen from the table, the maximum variation is for $\mathrm{E} 0$ at $\approx 0.8 \mu \mathrm{m}$ which can considered as minimal as it is within the measurement uncertainty determined using the droplet generator. Due to the resolution of the graduations of the glassware used to prepare the ethanol-gasoline blends, the average uncertainty in the ethanol content in gasoline is $\pm 2 \%$ for all the fuel blends.

\section{Results and discussion}

The number and volume distribution of the droplet diameters for all the fuel blends is shown in Fig. 10. It can be seen from

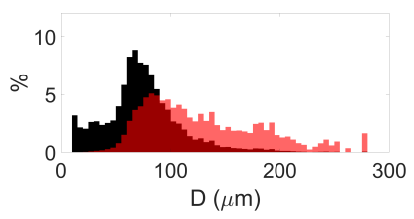

(a) E100

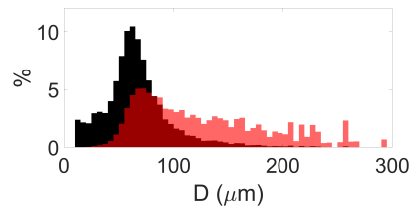

(c) $\mathrm{E} 60$

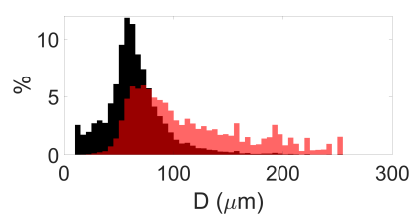

(e) $\mathrm{E} 40$

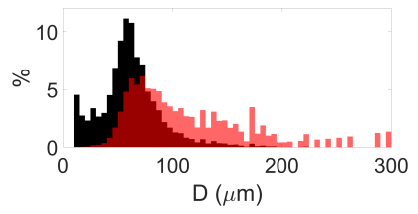

(g) E20

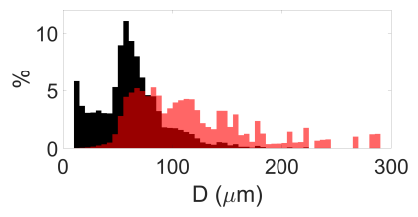

(i) $\mathrm{E} 10$

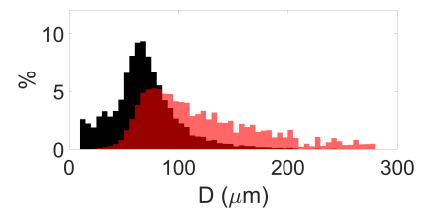

(b) $\mathrm{E} 80$

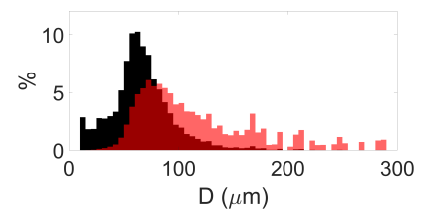

(d) $\mathrm{E} 50$

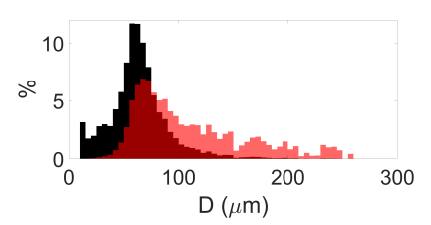

(f) E30

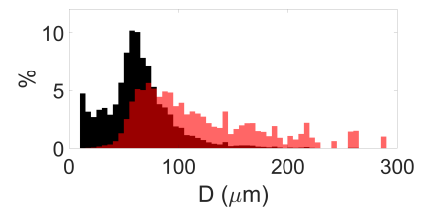

(h) E15

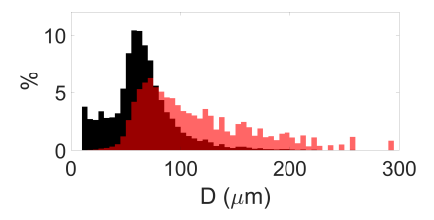

(j) E0
FIGURE 10. SUPERPOSED PROBABILITY DISTRIBUTIONS OF DROPLET NUMBER (BLACK) AND VOLUME (RED), EXPRESSED AS A PERCENTAGE OF OVERALL NUMBER AND TOTAL VOLUME RESPECTIVELY, FOR ALL FUEL BLENDS.

the figure that the number distribution for all the fuel blends are distinctly unimodal, with peak diameters of 60-70 $\mu \mathrm{m}$. Similar unimodal number distributions were found by Pan et al [7] when measuring direct injection gasoline spray with GSV. It can also be observed in the number distribution of all the fuel blends that there is a sharp peak of small droplets with diameters $10-15 \mu \mathrm{m}$. This is more significant for fuel blends E20, E15 and E10. This observation will be discussed in more detail later. For the determination of the droplet volume, the droplets were assumed to be perfectly spherical. The volume distribution is also unimodal, 
showing a sharp increase for the diameters up to $\approx 75 \mu \mathrm{m}$, followed by a gradual reduction in the total volume proportion.

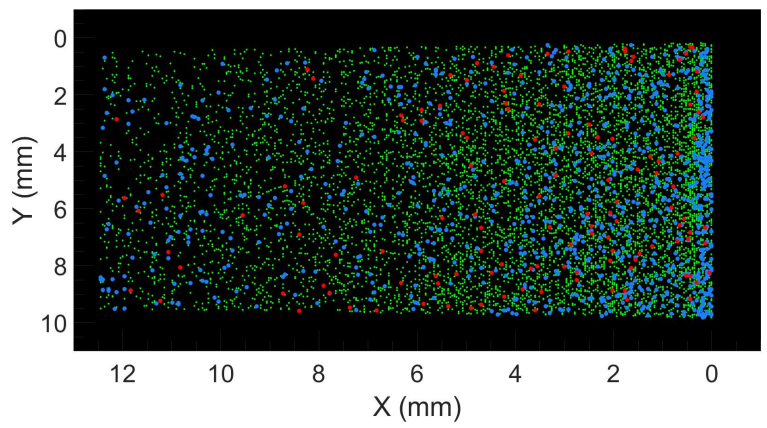

(a)

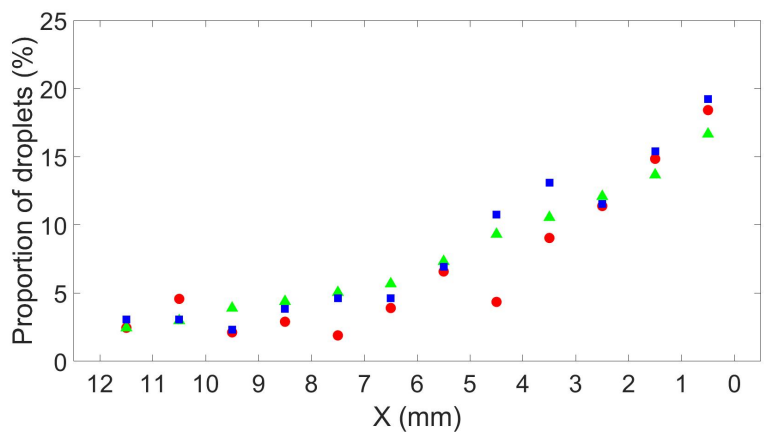

(b)

FIGURE 11. (a) DROPLET RADIAL (X) AND AXIAL (Y) POSITION WITH SIZE: $D_{10}<30 \mu \mathrm{m}$ (RED), $30 \mu \mathrm{m}<D_{10}<150$ $\mu \mathrm{m}$ (GREEN), $D_{10}>150 \mu \mathrm{m}$ (BLUE). (b) DROPLET PROPORTION WITH VARIATION IN RADIAL POSITION (X) FOR: $D_{10}<30 \mu \mathrm{m}$ (RED), $30 \mu \mathrm{m}<D_{10}<150 \mu \mathrm{m}$ (GREEN), $D_{10}>150 \mu \mathrm{m}$ (BLUE).

Figure 11(a) shows the spatial distribution of droplets, of three different droplet diameter ranges, within the ROI. Fig. 11(b) shows the change in droplet proportion with radial displacement. It can be seen that the three droplet size ranges are similarly distributed across the imaging window, showing the software processor does not have a spatial bias towards a particular diameter range. Based on this, the number distribution and the variation in droplet diameter was plotted, and has been shown in Figs. 12(a) and (b) respectively. It can be clearly seen from the figures that no significant change occurs in either the droplet frequency or diameter, with vertical displacement from the nozzle tip. However, both the droplet number and mean diameter show considerable variation with radial displacement from the spray centreline. The reduction in droplet number with increasing distance from the spray centreline is expected due to a reduction in the density of the spray, as has been observed by other re-

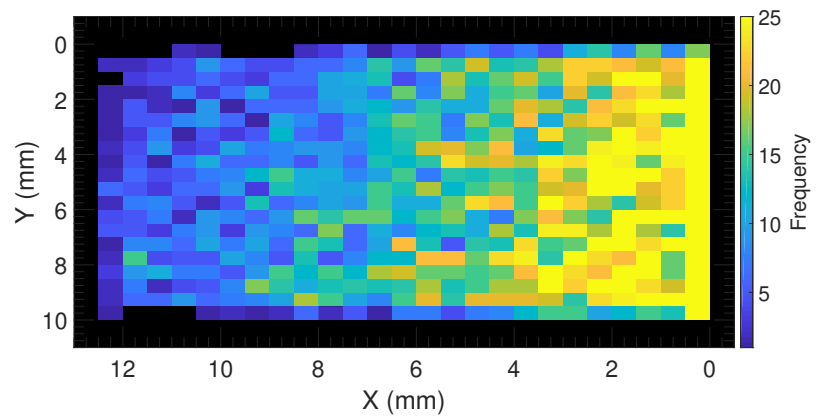

(a)

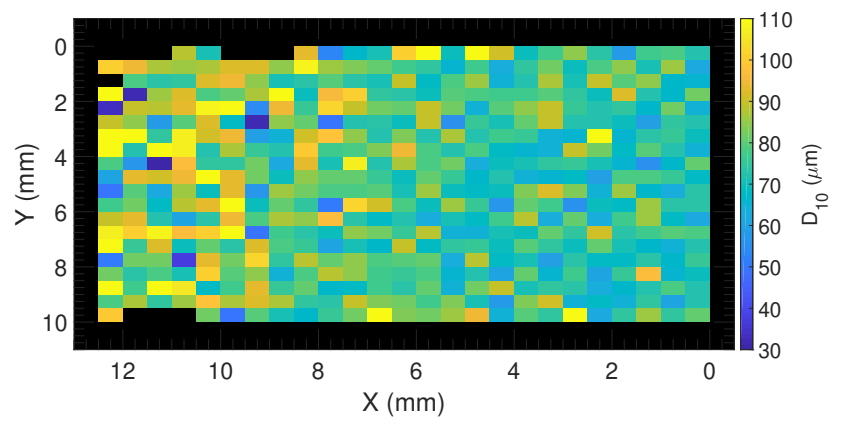

(b)

FIGURE 12. VARIATION IN (a) FREQUENCY AND (b) $D_{10}$, WITH AXIAL AND RADIAL DISPLACEMENT FROM THE NOZZLE.

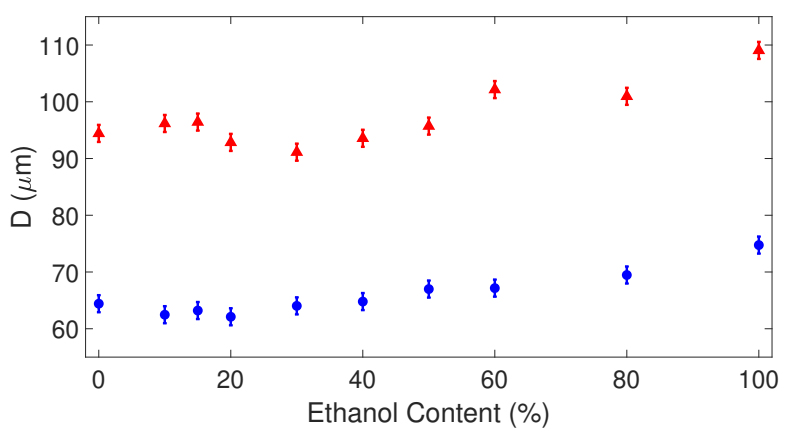

FIGURE 13. $D_{32}$ (RED) AND $D_{10}$ (BLUE) VALUES WITH ETHANOL CONTENT (\%).

searchers $[1,9]$. The droplet diameters show an increase with displacement from the centreline of the spray, which fits with the observations reported in literature $[1,9]$, and is speculated to be due to a reduction in droplet velocity and the evaporation of the smaller droplets near the fringe of the spray.

Fig. 13 shows the arithmetic mean diameter, $D_{10}$, and the SMD, $D_{32}$, with increasing ethanol content in gasoline. The results show that both $D_{10}$ and $D_{32}$ increased by $\approx 13 \%$ be- 


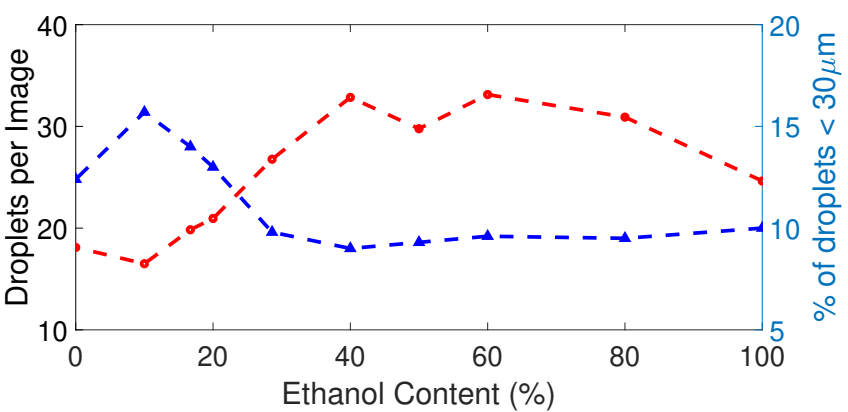

FIGURE 14. DROPLETS IDENTIFIED PER IMAGE (RED) AND PROPORTION OF DROPLETS BELOW $30 \mu \mathrm{m}$ (BLUE) FOR FUEL BLENDS.

tween E0 and E100, with fluctuating trends observed below $40 \%$ ethanol content, and a stronger positive correlation noted beyond $50 \%$ ethanol content in gasoline. The observed increase in droplet diameter with increasing ethanol proportion in gasoline has been reported in previous studies investigating fully developed ethanol-gasoline sprays $[5,8,13]$. The E60 shows a higher $D_{32}$ value than would be expected from the trend, this could be attributed to the presence of large diameter droplets $(<200 \mu \mathrm{m})$, as shown in Fig. 10(c). This effect is not apparent in the $D_{10}$ as larger droplet diameters have a greater impact on SMD calculations.

To further understand the observed trends for blends with less than $40 \%$ ethanol content, the analysed GSV oscillation pattern images were reviewed. A clear degradation in image quality was seen with decreasing ethanol content, being most pronounced in blends E20, E15, E10 and E0. Oscillation patterns became blurred and distorted, along with an increase in the occurrence of high frequency patterns attributable to multiple frequencies. Whilst the overall number of droplets did not significantly change with ethanol percentage, and the droplet density remained below 3000 droplets per cubic centimetre in all cases, the clarity of the patterns decreased.

This visual degradation in the quality of the analysed oscillation patterns has a noticeable impact on the number of droplet measurements per image, as shown in Fig. 14, with a substantial reduction in the number of detected droplets for blends containing less than $40 \%$ ethanol. A possible explanation for the degradation in image quality is the change in the colouration and opacity of the droplets with varying composition of the fuel blend. As the proportion of gasoline is increased, the blend becomes increasingly opaque and coloured. This pronounces the effect of off-axis attenuation (see Section 2.2) thus resulting in weaker signals detected by the camera sensor, reducing the signal to noise ratio. Due to this, the software processor is more likely to discard oscillation patterns, hence reducing the number of droplets detected per image.
Figure 14 also shows the proportion of droplets below 30 $\mu \mathrm{m}$ for the different fuel blends. It can be clearly observed from the figure that the proportion of droplets below $30 \mu \mathrm{m}$ increases as the ethanol content is reduced below 30\%. This increase was also observed in Fig. 10, where, particularly for E10, E15 and E20, a sharp peak in droplet numbers was seen for diameters 10$15 \mu \mathrm{m}$. The presence of low diameter droplets is potentially due to the erroneous detection and measurement of indistinct oscillation patterns, as described previously in Section 3. The reduction in image quality could further exacerbate the distortion of oscillation patterns, increasing the number of erroneous low diameter readings.

\section{Conclusions}

The TSI GSV system was used to acquire droplet measurements of sprays for a wide range of ethanol-gasoline blends, using a single hole PFI injector with an injection pressure of 3.5 bar. Measurements were taken in a $10 \mathrm{~mm} \times 12 \mathrm{~mm}$ viewing window (ROI) located $50 \mathrm{~mm}$ downstream from the injector nozzle tip and centred $20 \mathrm{~mm}$ radially from the spray centreline.

The GSV technique was found to be effective in measuring droplet diameters, with high repeatability, in liquid sprays with varying composition of ethanol and gasoline. The technique was able to provide a well-resolved spatial distribution of the number and size of droplets within the ROI. The GSV drop sizing measurements were validated using a mono-disperse droplet generator (Flow-focusing Mono-disperse Aerosol Generator, FMAG), and an excellent agreement (within 2\%) was observed.

For all ethanol-gasoline blends, distinct unimodal peaks between $\approx 60 \mu \mathrm{m}$ and $75 \mu \mathrm{m}$ were seen in all droplet size distributions. In blends with an ethanol content of $50 \%$ and more, increasing the proportion of ethanol in the fuel blend led to an increase in mean droplet diameter, as shown by both the $D_{10}$ and $D_{32}$ values. In fuel blends with less than $40 \%$ ethanol content there was a significant degradation in image quality, which reduced the number of droplets detected per image and increased erroneous identification of small diameter droplets. For all fuel blends, the concentration of droplets reduced with increasing radial distance from the spray centreline, while the mean droplet diameter increased.

Further investigations to understand the effects of droplet opacity and colouration on size measurements would be beneficial in developing interferometric techniques such as GSV for other bio-derived fuel comparisons.

\section{ACKNOWLEDGMENT}

The authors would like to acknowledge EPSRC (EP/P003036/1) for their financial support towards this work.

\section{REFERENCES}

[1] Pierson, S., Richardson, S., Rubini, P., Jermy, M., and Greenhalgh, D., 2000. "Laser characterization of a port fuel 
injector to provide boundary data for computational fluid dynamics". In IMechE Conference Transactions, Vol. 8, Citeseer, pp. 167-178.

[2] Ding, J.-W., Li, G.-X., and Yu, Y.-S., 2017. "The instability and droplet size distribution of liquid-liquid coaxial swirling spray: An experimental investigation". Experimental Thermal and Fluid Science, 82, pp. 166-173.

[3] Kannaiyan, K., and Sadr, R., 2014. "Experimental investigation of spray characteristics of alternative aviation fuels". Energy Conversion and Management, 88, pp. 1060-1069.

[4] Kim, W., Kim, H., and Yoon, W., 2012. "Experiments on atomization characteristics of the flash swirl spray". In 50th AIAA Aerospace Sciences Meeting including the New Horizons Forum and Aerospace Exposition, p. 176.

[5] Aleiferis, P., Behringer, M., OudeNijeweme, D., and Freeland, P., 2015. "Spray imaging and droplet sizing of sparkeroded and laser-drilled injectors with gasoline-butanol and gasoline-ethanol blends".

[6] Moriyoshi, Y., Uchida, R., Takagi, M., and Kubota, M., 2009. Numerical and experimental analyses of mixture formation process using a fan-shaped di gasoline spray: Examinations on effects of crosswind and wall impingement. Tech. rep., SAE Technical Paper.

[7] Pan, G., Shakal, J., Lai, W., Calabria, R., and Massoli, P., 2006. "Spray features investigated by gsv: A new planar laser technique". In Tenth International Conference on Liquid Atomization and Spray Systems (ICLASS), Kyoto, Japan, Aug.

[8] Park, S. H., Kim, H. J., Suh, H. K., and Lee, C. S., 2009. "Atomization and spray characteristics of bioethanol and bioethanol blended gasoline fuel injected through a direct injection gasoline injector". International Journal of Heat and Fluid Flow, 30(6), pp. $1183-1192$.

[9] Feng, Z., Zhan, C., Tang, C., Yang, K., and Huang, Z., 2016. "Experimental investigation on spray and atomization characteristics of diesel/gasoline/ethanol blends in high pressure common rail injection system". Energy, 112, pp. 549-561.

[10] Li, T., Nishida, K., and Hiroyasu, H., 2011. "Droplet size distribution and evaporation characteristics of fuel spray by a swirl type atomizer". Fuel, 90(7), pp. 2367-2376.

[11] Dehaeck, S., and Van Beeck, J., 2008. "Multifrequency interferometric particle imaging for gas bubble sizing". Experiments in fluids, 45(5), p. 823.

[12] Duan, H., Romay, F., Liu, B., and Naqwi, A., 2016. "Flowfocusing monodisperse aerosol generator for calibration of spray diagnostics instruments". In 18th International Symposium on the Application of Laser and Imaging Techniques to Fluid Mechanics, Lisbon, Portugal.

[13] Fajgenbaum, R., and dos Santos, R. G., 2016. "Influence of fuel temperature on atomization parameters in a pressureswirl atomizer from a port fuel injector by shadowgraphy technique". Journal of the Brazilian Society of Mechanical Sciences and Engineering, 38(7), pp. 1877-1892.

\section{Appendix}
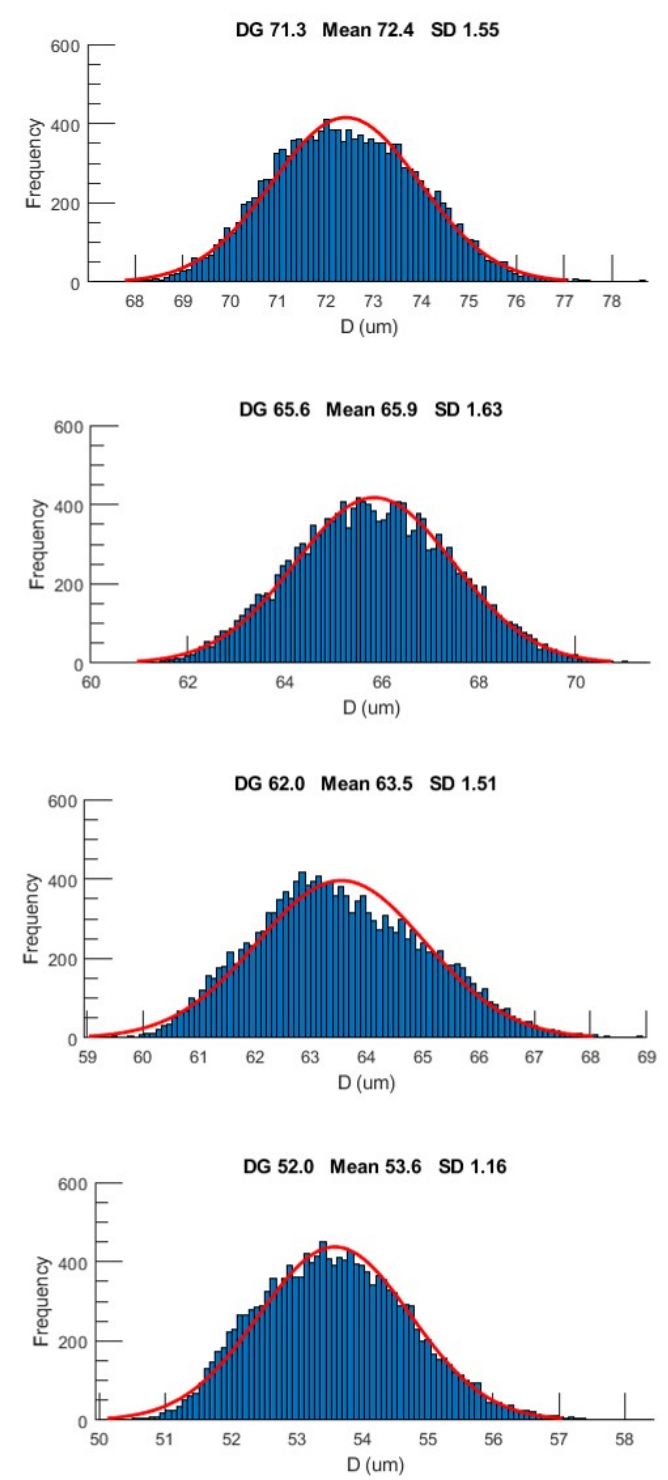

FIGURE A.1. FREQUENCY DISTRIBUTION OF FMAG DROPLETS MEASURED BY GSV, CORRESPONDING TO TAB.2 $($ DG $=$ SET FMAG DROPLET DIAMETER, $S D=$ STANDARD DEVIATION) 